\title{
Traduire
}

Revue française de la traduction

$227 \mid 2012$

Éco, socio, philo... \& co

\section{Traduire dans le domaine de l'économie écologique : les difficultés terminologiques}

Sabri-Fabrice Sayhi

\section{OpenEdition}

Journals

Édition électronique

URL : http://journals.openedition.org/traduire/474

DOI : $10.4000 /$ traduire.474

ISSN : 2272-9992

Éditeur

Société française des traducteurs

Édition imprimée

Date de publication : 15 décembre 2012

Pagination : $35-46$

ISSN : 0395-773X

\section{Référence électronique}

Sabri-Fabrice Sayhi, «Traduire dans le domaine de l'économie écologique : les diffıcultés

terminologiques », Traduire [En ligne], 227 | 2012, mis en ligne le 01 décembre 2014, consulté le 30 avril 2019. URL : http://journals.openedition.org/traduire/474 ; DOI : 10.4000/traduire.474 


\section{Traduire dans le domaine de l'économie écologique : les difficultés terminologiques}

\section{Sabri-Fabrice Sayhi}

La traduction dans le domaine des sciences sociales est un exercice complexe qui demande des connaissances transversales dans différents domaines. L'on pourrait rétorquer que cette caractéristique est commune à tous les types de traductions scientifiques; toutefois, cela se vérifie particulièrement en sciences sociales. Par ailleurs, la traduction de ce type de textes présente la spécificité suivante : bien que les sciences sociales semblent de prime abord plus abordables que les sciences " dures ", car elles sont plus directement liées aux êtres humains et à la vie quotidienne, ce domaine recèle de multiples difficultés.

Une science sociale s'oppose intrinsèquement à une science " exacte " par sa nature épistémologique. La divergence d'opinions est donc encore plus importante que dans les sciences " dures ", ce qui a bien sûr une incidence sur la traduction et la terminologie. En particulier, lorsqu'une science sociale est nouvelle, elle présente d'autant plus de difficultés pour le traducteur que sa terminologie et ses définitions font rarement l'unanimité chez les spécialistes, surtout si elle est composée de plusieurs autres sciences. En outre, le problème de la néologie peut également se présenter et il arrive souvent que certains termes utilisés dans une science sociale donnée soient les mêmes que dans d'autres sciences, avec une acception différente.

L'économie écologique(1) est l'exemple le plus frappant de ce qui vient d'être énoncé ci-dessus. En effet, cette science qui constitue une sorte de macro-domaine englobant bon nombre d'autres sciences peut sembler de prime abord compréhensible au néophyte qui serait amené à traduire un texte traitant d'économie écologique. Mais la complexité sous-jacente qui la caractérise apparaît dès les premières recherches documentaires. En guise de première définition, on peut dire de l'économie écologique qu'il s'agit d'une tentative de réconciliation du développement économique et de l'environnement en vue de préserver les ressources naturelles et de pourvoir équitablement aux besoins de la société.

(1) Cet article est tiré de mon mémoire de Master 2 Industrie de la langue et traduction spécialisée (Université Paris 7 Diderot) sur la traduction et la terminologie dans le domaine de l'économie écologique (septembre 2012). À ce titre, je souhaite remercier mes directeurs de mémoire Débora Farji-Haguet et Geneviève Bordet pour leur soutien, ainsi que John Humbley qui faisait partie du jury de soutenance. 


\section{1) État de la recherche}

Il est en premier lieu intéressant de se pencher sur le paysage documentaire de l'économie écologique. Étant donné qu'il s'agit d'une science sociale transdisciplinaire, les spécialistes de ce champ d'étude présentent des profils très variés. lls sont géographes, biologistes, spécialistes en études environnementales, agronomes, philosophes ou sociologues. II s'agit aussi bien de scientifiques travaillant sur le terrain que d'universitaires; des militants et des ONG travaillent aussi très souvent en étroite collaboration avec les chercheurs. Ce foisonnement d'experts de tous horizons a pour avantage de conférer à ce domaine un certain pluralisme, censé contrecarrer la vision univoque de l'économie néoclassique à laquelle s'oppose l'économie écologique.

La plupart des articles qui portent sur le thème de l'économie écologique sont rédigés en anglais, même par les francophones. II est tout de même possible de trouver de la documentation en français : il s'agit, le plus souvent, d'articles scientifiques rédigés par des chercheurs, soit sur la théorie de l'économie écologique, avec beaucoup d'articles de type " état de l'art ", soit des articles de recherche sur l'économie écologique appliquée à une certaine activité économique ou à un certain écosystème du monde.

La recherche en économie écologique n'a pris de l'importance en France que depuis une vingtaine d'années avec, entre autres, René Passet, Jean-Paul Deléage ou Fabrice Flipo.

Pour la vulgarisation, on peut trouver des articles dans des journaux tels que Le Monde diplomatique ou Le Figaro. Les articles scientifiques sont généralement disponibles sur Internet, mais également sur papier. En français, on en trouve notamment dans les revues Développement durable et territoire, Écologie et politique ou encore Etopia (publiée par un centre de recherche en écologie politique basé en Belgique). Toutefois, on constate qu'il n'existe pas de revue propre à l'économie écologique dans le monde francophone.

On trouve aussi quelques ouvrages spécialisés sur l'économie écologique : citons l'un des ouvrages phares de Nicholas Georgescu-Roegen, auteur qui fait référence en la matière, La décroissance, entropie, économie, écologie(2), Le Développement soutenable de FranckDominique Vivien et parmi les ouvrages à vocation vulgarisatrice, L'économique et le vivant de René Passet.

Sont également disponibles des rapports d'activité s'adressant aux spécialistes, comme le Manuel du CEECEC(3) qui est complété par le seul glossaire de l'économie écologique proposé à ce jour. Dans la même lignée, citons le projet du TEEB(4) dont le rapport d'activités est

(2) Traduit de l'anglais par Jacques Grinevald et Ivo Rens.

(3) Civil Society Engagement with Ecological Economics (Engagement de la société civile avec l'économie écologique). Fruit de la collaboration d'ONG et de chercheurs en économie écologique, ce projet a été financé par la Commission européenne et présente différentes études de cas pratiques.

(4) The Economics of Ecosystems and Biodiversity (l'Économie des écosystèmes et de la biodiversité), projet dont l'équipe est constituée de manière similaire au projet CEECEC, également financé par la Commission européenne. 
disponible en français et en espagnol. On remarque néanmoins que ces rapports, fruits de la collaboration de plusieurs chercheurs, ont d'abord été rédigés en anglais, pour ensuite faire l'objet de traductions vers le français ou l'espagnol.

Enfin, il existe plusieurs sites Internet et articles publiés dans des revues militantes radicales, qui s'opposent au schéma traditionnel de la croissance et remettent en question le développement aveugle des pays développés au détriment des plus démunis. Ceux-ci s'adressent davantage au grand public. S'y ajoutent des revues papier également disponibles en ligne telles que Mouvements, Mauss(5) ou encore Silence qui se focalisent sur l'aspect politique de l'économie écologique et se caractérisent par la virulence de leurs articles.

En conclusion, l'on peut dire que si certains auteurs rédigent des ouvrages ou des articles sur le thème de l'économie écologique (ou l'un de ses aspects), la documentation dans ce domaine est peu fournie. II est d'ailleurs à noter qu'il n'existe pas de revue francophone dédiée spécifiquement à l'économie écologique, alors que les anglophones disposent de la leur : Ecological Economics. D'où la difficulté de trouver des équivalents en langue française des termes nés en anglais, comme c'est souvent le cas en science, notamment pour les sciences nouvelles. Pourtant, il n'en va pas tout à fait de même pour l'espagnol : en effet, bien que les hispanophones ne disposent pas non plus d'une revue consacrée à l'économie écologique, l'Espagne (plus précisément la Catalogne) possède un centre de recherche dans ce domaine, I'" Institut de ciència i tecnologia ambientals ", qui est plus important que son homologue français. Cette caractéristique, ajoutée aux nombreux auteurs latino-américains qui publient également sur ce sujet, facilite les recherches documentaires et terminologiques en espagnol.

\section{2) "Soutenabilité " et changement de paradigme}

L'économie écologique est une science sociale transdisciplinaire qui propose une nouvelle vision de la relation entre économie et environnement. Elle considère l'économie comme un sous-système de l'écosystème mondial et cherche à évaluer la " soutenabilité " des échanges entre ces deux ensembles grâce à une analyse multicritères. Ces critères comprennent notamment des éléments de l'économie et de l'écologie en général, mais aussi des éléments de l'écologie industrielle, de l'écologie politique, de la physique, de la biologie, de la géologie, de la sociologie, de l'anthropologie et de la philosophie.

D'un point de vue traductologique, le premier terme qui pose problème est le néologisme " soutenable ", ainsi que son dérivé nominal " soutenabilité ". L'adjectif " soutenable ", traduction du terme sustainable en anglais, ou encore sostenible en espagnol, ne peut en aucun cas être traduit par " durable " même si cette traduction est attestée et généralement utilisée

(5) Revue du Mouvement anti-utilitariste en sciences sociales. 
en sciences environnementales. En effet, les partisans de l'économie écologique francophones considèrent que " durable " est une mauvaise traduction de l'adjectif sustainable; ce dernier est censé indiquer que l'activité économique doit pouvoir être " supportée " par l'environnement, ou " soutenue ". "Soutenable " serait donc plus à même de traduire l'idée de viabilité de l'économie, avec la volonté de s'opposer à la doctrine du "développement durable ". Ce concept à la mode est vu par les économistes écologiques comme une justification du capitalisme et comme un argument marketing visant à prolonger l'exploitation des ressources sans vrai changement de comportement. Les spécialistes du domaine refusent donc d'accoler l'adjectif " durable " au terme " développement ", car ils remettent en question la pérennité illusoire de l'exploitation des ressources que sous-tend la notion de "développement durable ".

Pourtant, l'économie écologique et le développement durable poursuivent un objectif commun : prendre en compte l'ensemble des générations actuelles et des générations à venir dans le développement économique. Mais les économistes écologiques, estimant que cet objectif n'a pas été atteint par le " développement durable ", prônent une prise en compte des limites environnementales et sociales de l'économie beaucoup plus stricte, qui se traduit par un glissement terminologique en français. Il est intéressant de constater que ni le terme anglais (sustainable) ni le terme espagnol (sostenible) ne changent d'un domaine à l'autre, alors que la radicalisation du concept a eu pour conséquence une transformation non seulement du signifié, mais également du signifiant en français. Le traducteur francophone chargé de traduire un texte lié aux sciences environnementales doit donc choisir entre les termes " durable " et " soutenable ", indiquant son adhésion aux idées de l'économie écologique s'il opte pour le second. Or, l'anglais et l'espagnol restent plus neutres puisqu'un seul et même terme est disponible dans chacune des deux langues.

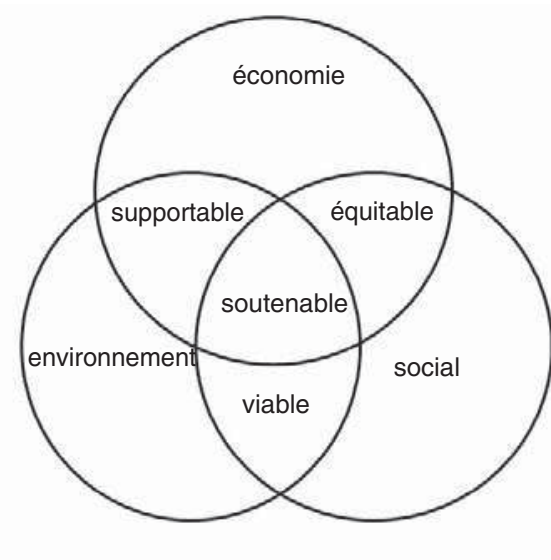

Soutenabilité faible

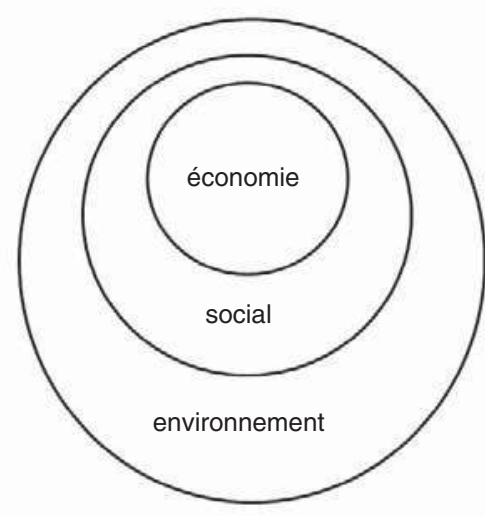

Soutenabilité forte

Source : MARÉCHAL Aurélie, 2011, "Économie écologique : principes de base ", dans Etopia n 8 , http://www.etopia.be/spip.php?article1741, consulté le 28 octobre 2011. 
L'économie écologique remet en cause le fameux schéma du développement durable du rapport Brundtland(6), qui semble indiquer que les trois sphères (économique, environnementale et sociale) qui le composent sont, au moins en partie, autosuffisantes, même si elles s'entrecroisent. En effet, les sphères économique et sociale apparaissent comme une sorte de "génération spontanée " dans le schéma traditionnel, puisqu'une partie de chacune d'elles n'a aucun contact avec la sphère environnementale. Les économistes écologiques préfèrent concevoir ces trois sphères comme des poupées russes qui s'imbriquent l'une dans l'autre : la sphère économique est vue comme un sous-ensemble de la sphère sociale dont elle dépend, qui ellemême est un sous-ensemble de l'environnement dont elle dépend à son tour.

\section{3) Une terminologie caractérisée par la métaphore}

Comme nous venons de le voir, l'économie écologique se caractérise par un changement de paradigme ; sa terminologie en est d'ailleurs révélatrice. En effet, bon nombre de termes du domaine sont des métaphores, des représentations qui renvoient à cette nouvelle vision de la nature : " métabolisme social ", " empreinte hydrique ", " passif environnemental ", " dette écologique ", par exemple. On remarque de prime abord un important usage de l'" empreinte " et de la " dette ". Ce recours à la métaphore pédagogique de l'" empreinte ", créée en 1992 par Mathis Wackernagel et William Rees en tant qu'outil censé évaluer la pression exercée par un groupe sur un autre groupe ou une zone(7), est très répandu depuis quelques années dans les sciences sociales. En ce qui concerne la métaphore de la " dette ", celle-ci au même titre que celle du " passif environnemental ", est révélatrice de la métaphore économique de l'écologie qui caractérise ce champ d'étude. Toutefois, il serait erroné de penser que cette métaphore vise simplement à concevoir l'environnement en termes économiques. II s'agit davantage d'une réconciliation de ces deux domaines visant à mettre en lumière leur indissociabilité. En effet, la " dette écologique " n'exclut pas l'obtention d'une compensation pécuniaire destinée aux populations démunies qui souffrent le plus directement des dégâts environnementaux. Mais au-delà de sa dimension socio-économique, cette métaphore vise avant tout à mettre en exergue la crise écologique : son objectif est de montrer que l'économie génère un autre type de dettes, tout aussi graves si ce n'est plus, que les dettes financières. II en va de même pour les termes " capital naturel " et " biens et services environnementaux ", qui n'attribuent pas une valeur strictement mercantile aux ressources de la nature mais montrent que celle-ci peut également être vue comme une forme de capital d'une importance majeure, puisqu'elle est la condition sine qua non de la création des autres formes de capital par les humains. Les éco-

(6) Commission mondiale sur l'environnement et le développement de l'ONU, 1988, Rapport Brundtland, Notre avenir à tous, Montréal, Éditions du Fleuve.

(7) DUCHEMIN Eric, 2001, "Mathis Wackernagel et William Rees, notre empreinte écologique ", dans Vertigo, http://vertigo.revues.org/5230, consulté le 4 novembre 2012. 
nomistes écologiques utilisent d'ailleurs ces derniers termes avec beaucoup de précaution et de façon beaucoup plus euristique, craignant que leur usage ne mène à une « monétarisation de la nature „(8). En ce sens, les économistes écologiques s'opposent ainsi aux partisans d'une science voisine, l'économie environnementale.

\section{4) Économie écologique vs économie environnementale}

Il est intéressant de noter que dans plusieurs termes de l'économie écologique (" justice environnementale ", " empreinte environnementale ", " dette écologique "), l'adjectif " écologique " peut se substituer à " environnemental " et vice versa, sans incidence sur le sens du terme. Pourtant, il existe une exception et pas des moindres : l'économie écologique et l'économie environnementale. II est essentiel de distinguer ces deux domaines sur le plan terminologique, ce qui n'est pas chose aisée et pour cause : certains économistes écologiques considèrent qu'ils vont de pair. Toutefois, il existe une différence fondamentale entre ces deux champs d'étude. Alors que l'économie environnementale se contente d'affecter une valeur monétaire à l'environnement afin d'évaluer les conséquences de l'économie sur la nature, l'économie écologique va plus loin en s'efforçant d'utiliser des indicateurs environnementaux dits " indicateurs biophysiques " mais également des indicateurs sociaux. Elle est davantage intéressée par le rendement énergétique de l'exploitation des ressources naturelles et cherche à accroître ce rendement tout en limitant la pression exercée sur les ressources naturelles et les conséquences sociales négatives qui découlent du développement économique. C'est pourquoi ses adeptes développent de nouveaux indicateurs autres que l'indicateur financier.

Cela dit, il serait tout à fait erroné de considérer la frontière entre ces deux paradigmes comme hermétique. En effet, bien que bon nombre d'économistes écologiques soient réticents à l'idée d'accorder un rôle dominant à l'indicateur monétaire, la majorité d'entre eux conçoivent qu'il peut s'avérer nécessaire d'inclure cet indicateur dans l'évaluation de la " soutenabilité " économique, à côté d'autres indicateurs, ne serait-ce que pour impressionner l'opinion publique et changer les tendances. II est à noter que la notion d'indicateur est extrêmement répandue dans les sciences sociales et constitue un invariant de ce type de sciences.

Ainsi, il est parfois délicat de délimiter la terminologie de l'une et de l'autre science, étant donné que certains termes sont parfois utilisés dans les deux, tel que le "capital naturel " mentionné ci-dessus, avec un sens néanmoins différent. En économie écologique, le capital économique ne peut en aucun cas se substituer au " capital naturel ", alors qu'en économie environnementale, le premier peut compenser le second (voir schéma). Ce type de distinctions doit absolument être pris en considération par le traducteur pour une compréhension optimale du texte source.

(8) MERINO-SAUM Albert et al., 2012, "Que peut-on apprendre de l'économie écologique ? ", dans La vie des idées, http://www.laviedesidees.fr/Que-peut-on-apprendre-de-l.html, consulté le 6 avril 2012. 


\section{5) Une terminologie teintée idéologiquement qui ne fait pas consensus}

Il existe donc des divergences entre spécialistes de l'économie environnementale et spécialistes de l'économie écologique, mais également au sein même de cette dernière, qui se caractérise par un grand pluralisme d'opinions. Cela est en partie dû au fait que l'écologie politique, sousdomaine de l'écologie, tient une part importante dans l'économie écologique. Cette position se reflète dans les termes qu'elle lui emprunte, comme " justice environnementale ", "exportation de déchets toxiques " ou encore la fameuse " décroissance ". Ces exemples montrent qu'il s'agit d'une terminologie particulièrement connotée politiquement, car elle reflète une volonté de dénonciation et de revendication. En particulier le terme " décroissance ", qui ne fait pas consensus parmi les experts du domaine. En effet, bon nombre d'économistes écologiques rebutés par le côté très provocateur et subversif de ce terme lui préfèrent le terme " acroissance " (agrowth en anglais et acrecimiento en espagnol). Cela dit, l'on peut objecter que même si le terme de "décroissance " semble de prime abord plus radical, car on pourrait penser qu'il prône une croissance négative, alors que l'ı acroissance " ne préconiserait qu'une absence de croissance, il est difficile de définir l'un ou l'autre terme dans la pratique. Comme l'explique Serge Latouche tout au long de son ouvrage Petit traité de la décroissance sereine, il s'agit en réalité de quasi-synonymes qui traduisent avant tout une remise en question du modèle de croissance imposé par le système capitaliste, de la part des "objecteurs de croissance ".

Le terme " décroissance " illustre une fois de plus la transdisciplinarité de ce domaine qui divise les chercheurs et empêche d'avoir une vision univoque du domaine : certains voulant rester très scientifiques rejettent ce terme pourtant utilisé par un grand nombre de chercheurs qui n'ont pas peur d'exhiber leurs couleurs politiques, l'écologie politique étant très nettement marquée par une vision de gauche. Cela a donc une incidence sur le travail du traducteur qui, d'une certaine manière prend parti lorsqu'il traduit et décide de jeter son dévolu sur tel ou tel terme.

\section{6) Une terminologie qui cache un sens nouveau}

S'il existe des divergences sur les plans scientifique, idéologique et terminologique comme nous venons de le voir, cela est notamment dû au pluralisme de ce domaine. En effet, les économistes écologiques proviennent de différents horizons scientifiques. De plus, la plupart des termes de cette science sont des apports d'autres sciences et rares sont les termes qui sont propres à l'économie écologique. On constate d'ailleurs qu'il résulte de ce " pot-pourri terminologique " des variations sémantiques pour certains des termes utilisés.

Prenons l'exemple du terme " externalité ». Dans le domaine de l'économie, celui-ci fait référence aux conséquences non volontaires, positives ou négatives d'une activité économique. 
Mais les tenants de l'économie écologique, qui s'intéressent avant tout aux externalités négatives, vont plus loin dans la définition de celles-ci et les considèrent comme des " coûts transférés vers les plus faibles "(9), s'opposant ainsi à l'attitude de déresponsabilisation traditionnellement associée à ce terme. Ainsi, pour les économistes écologiques, les externalités négatives ne consisteraient qu'en une économie de dépenses avec de graves conséquences socio-environnementales, car elles touchent généralement les plus démunis (qui sont ceux qui dépendent le plus directement de la nature).

Un autre exemple digne d'intérêt est le terme " incommensurabilité ". On remarque tout d'abord que le mot « incommensurable » s'utilise dans la langue générale ; ainsi, il est facile pour le traducteur novice de passer à côté de ce qui s'avère être un terme doté d'un sens bien précis, avec d'éventuelles conséquences sur l'interprétation du texte source et la formulation du texte cible. C'est d'ailleurs l'une des difficultés principales de la traduction dans le monde des sciences sociales en général et, en particulier, dans le domaine de l'économie écologique (on peut d'ailleurs citer le terme " décroissance " comme autre exemple). L'adjectif « incommensurable ", bien qu'il soit utilisé dans la langue générale est, en réalité, également un terme utilisé en sciences, qui désigne l'impossibilité de comparer deux valeurs ayant une unité de mesure différente. En économie écologique, ce principe de base est appliqué au domaine du développement économique en relation avec l'environnement : il s'agit de l'idée selon laquelle il est difficile (voire contre nature) d'évaluer la biosphère en termes uniquement monétaires puisque les services qu'elle apporte sont difficilement chiffrables(10).

\section{7) Concurrence}

Un autre grand problème auquel se trouve confronté le traducteur en économie écologique est le foisonnement de concurrents (synonymes et quasi-synonymes). Une fois de plus, ce phénomène est récurrent en sciences sociales, car ce qui les différencie des sciences dites "dures " est leur plus grande flexibilité sur le plan théorique, qui a bien entendu des conséquences sur le plan terminologique. En outre, ce phénomène est d'autant plus présent dans les sciences nouvelles et de surcroît transdisciplinaires : la recherche est en plein développement, les spécialistes affluent, les théories fusent et la terminologie n'est donc pas figée.

Ainsi, en économie écologique il n'est pas rare de trouver plusieurs termes équivalents pour le même signifié, aussi bien dans la langue source qu'en français. Cela pose bien évidemment des problèmes de traduction de différentes natures. Premièrement, le traducteur soucieux

(9) MARTínEZ ALIER Joan, 2009, " Hacia un decrecimiento sostenible en las economías ricas ", dans Revista de economía crítica, $n^{\circ}$ 8, http://www.ecologiaradical.com.mx/VB/Biblioteca/Hacia un Decrecimiento Sostenible en las Economías Ricas.pdf, consulté le 10 septembre 2011.

(10) MERINO-SAUM Albert et al., Op. cit., p 6. 
d'employer le terme le plus usité dans sa langue dans le domaine de spécialité doit constituer un corpus afin de savoir quel est le terme le plus récurrent. On peut citer l'exemple de l'" empreinte hydrique(11) " également désignée par les termes " empreinte eau ", " empreinte de l'eau ", " empreinte sur l'eau " ou encore " empreinte hydrologique ".

En outre, le traducteur peut être dérouté par la profusion de ces synonymes, tant dans la langue source que dans la langue cible. II peut être amené à se demander s'il s'agit de concepts différents, comme dans le cas du " capital naturel ", pour lequel on trouve les équivalents " capital environnemental " ou encore " capital écologique ".

Ce phénomène touche surtout les termes composés. En outre, au sein de certains de ces termes composés, il est fréquent qu'un mot soit remplacé par un mot voisin : parfois un synonyme, parfois un quasi-synonyme, parfois ni l'un ni l'autre. Une fois de plus, il est difficile de savoir lequel est le terme de référence. Ainsi, pour le terme " analyse des flux de matière ", on trouve aussi " évaluation des flux de matière " et même " comptabilisation des flux de matière ". En ce qui concerne le terme " exportation de déchets toxiques ", les quasi-synonymes suivants sont également utilisés : " déversement de déchets toxiques " et " transfert de déchets toxiques". Dans ce dernier exemple, le mot qui change au sein des concurrents (" exportation " devient " déversement " ou " transfert ") n'est même pas un quasi-synonyme du mot qui compose le terme de référence, mais le sens global du terme composé reste le même.

\section{8) Utilisation de sigles anglo-saxons}

Pour certains termes, tels qu' " analyse des flux de matière " ou encore " appropriation humaine de la production primaire nette ", le traducteur est confronté au dilemme suivant : en consultant la littérature scientifique française du domaine, on remarque que les scientifiques francophones étant très influencés par l'anglais, n'hésitent pas à utiliser l'acronyme des termes anglais dans leurs textes. Par exemple: HANNP (Human Appropriation of Net Primary Production) et MFA (material flow analysis). Le traducteur doit alors opérer un choix. II peut suivre une approche normative et considérer que cet usage de l'acronyme anglo-saxon est abusif et qu'il serait préférable que les chercheurs optent pour un terme (ou un sigle) français ; il choisit donc de chercher et d'utiliser le terme traduit. Mais il peut également opter pour une approche descriptive et considérer que si c'est l'acronyme anglais qui est le plus souvent utilisé, il convient d'utiliser celui-là afin de ne pas dérouter les lecteurs.

(11) Indicateur du " volume total de l'eau de pluie, des eaux de surface et des eaux souterraines mobilisées pour créer les biens et services utilisés par la population, ainsi que les eaux usées déversées dans la nature suite à une telle production " (GNEHM Felix, 2012, Étude de l'empreinte hydrique suisse, https://www.deza.admin.ch/ressources/resource_fr_209748.pdf, consulté le 9 mai 2012.) 


\section{9) Conclusion}

Cet article a tenté de montrer que la traduction de textes liés à l'économie écologique et, de façon plus générale, aux sciences sociales est un exercice complexe qui se heurte à divers obstacles, en particulier lorsqu'il s'agit d'une science nouvelle.

Comme nous avons pu le voir, la difficulté principale que représente la traduction en économie écologique est d'ordre terminologique. En effet, la littérature scientifique et les glossaires en langue française n'abondent pas, ce qui complique la recherche d'équivalents dans la langue cible pour le traducteur francophone. Par ailleurs, bien que les termes techniques semblent parfois directement compréhensibles, ceux-ci cachent souvent un sens bien précis et des connotations insoupçonnées. Certains termes peuvent aussi ne pas faire consensus entre spécialistes, ou revêtir un sens différent par rapport au sens qu'ils ont dans des champs voisins ou dans la langue courante. Enfin, le traducteur est amené à faire face au phénomène de la synonymie, problème typique des sciences jeunes.

D'ailleurs, la spécificité la plus saillante des sciences novatrices est leur propension à la néologie. En effet, ces sciences sociales nouvelles bousculent les idées reçues et remplacent les concepts obsolètes. Comme nous le savons bien, culture et langues sont intimement liées ; ainsi lorsque les concepts évoluent, les noms évoluent avec eux et s'installent petit à petit dans le langage : hier encore, nous parlions de "développement durable ", demain nous parlerons de "décroissance soutenable ".

sfsayhi@gmail.com

Sabri-Fabrice SAYHI, originaire de la ville de Nice, a commencé par faire des études d'anglais à l'université de Nice Sophia-Antipolis. Après avoir obtenu son master recherche en Études anglophones, il se tourne vers des études de français langue étrangère et enseigne le français, d'abord à Nice, puis au Royaume Uni, en Espagne et au Mexique dans différentes institutions. II s'oriente ensuite vers la traduction, discipline à laquelle il s'était intéressé en particulier lors d'un stage dans une agence de traduction dans le cadre de son M2 recherche. II décide alors de s'inscrire en master 2 Industrie de la langue et traduction spécialisée à l'université Paris 7 Diderot. Son mémoire porte sur la traduction dans les sciences sociales, plus particulièrement l'économie écologique. II travaille en parallèle pour l'ONG internationale CARE France en tant que traducteur technique, ce qui l'amène à se spécialiser dans le domaine des sciences socio-environnementales. 


\section{Glossaire des termes les plus récurrents de l'économie écologique(12)}

\begin{tabular}{|c|c|c|}
\hline Anglais & Espagnol & Français \\
\hline agrowth & acrecimiento & acroissance \\
\hline biophysical indicator & indicador biofísico & indicateur biophysique \\
\hline biopiracy & biopiratería & biopiraterie \\
\hline carbon debt & deuda de carbono & dette carbone \\
\hline degrowth & decrecimiento & décroissance \\
\hline depletion of natural resources & agotamiento de los recursos naturales & épuisement des ressources naturelles \\
\hline ecological debt & deuda ecológica & dette écologique \\
\hline ecological footprint & huella ecológica & empreinte écologique \\
\hline entropy & entropía & entropie \\
\hline environmental good & bien ambiental & bien environnemental \\
\hline environmental justice & justicia ambiental & justice environnementale \\
\hline environmental liability(13) & pasivo ambiental & passif environnemental \\
\hline environmental service & servicio ambiental & service environnemental \\
\hline exosomatic & exosomático & exosomatique \\
\hline export of toxic waste & exportación de residuos tóxicos & exportation de déchets toxiques \\
\hline externality & externalidad & externalité \\
\hline $\begin{array}{l}\text { human appropriation of net } \\
\text { primary production }\end{array}$ & $\begin{array}{l}\text { apropiación humana de la producción } \\
\text { primaria neta }\end{array}$ & $\begin{array}{l}\text { appropriation humaine de la production } \\
\text { primaire nette }\end{array}$ \\
\hline incommensurable & inconmensurable & incommensurable \\
\hline material flow analysis & análisis de los flujos de materiales & analyse des flux de matière \\
\hline natural capital & capital natural & capital naturel \\
\hline relational good & bien relacional & bien relationnel \\
\hline social metabolism & metabolismo social & métabolisme social \\
\hline sustainable & sostenible & soutenable \\
\hline water footprint & huella hídrica & empreinte hydrique \\
\hline
\end{tabular}

(12) Comme nous l'avons vu précédemment, la terminologie de l'écologie économique se caractérise par une importante synonymie. Ainsi, les termes qui apparaissent dans ce glossaire sont ceux qui sont le plus fréquemment utilisés dans le domaine d'étude.

(13) Ce terme peut également signifier " responsabilité environnementale " en français, selon le contexte. 


\section{Bibliographie}

GEORGESCU-ROEGEN Nicholas, 1995. La décroissance. Entropie - Écologie - Économie, nouvelle édition, (première édition : 1979), présentation et traduction de MM. Jacques Grinevald et Ivo Rens, Paris, Sang de la terre.

VIVIEN Frank-Dominique, 2005. Le développement soutenable, Paris, La découverte.

PASSET René, 1979. L'économique et le vivant, Paris, Payot.

LATOUCHE Serge, 2007. Petit traité de la décroissance sereine, Paris, Mille et une nuits.

MERINO-SAUM Albert et al., 2012. "Que peut-on apprendre de l'économie écologique ?", dans La vie des idées, http://www.laviedesidees.fr/Que-peut-on-apprendre-de-I.html, consulté le 6 avril 2012.

BONAÏUTI Mauro, 2002. "À la conquête des biens relationnels ", dans Silence n 280, http://kropot.free.fr/Silence-decroissance.htm\#SOMMAIRE, consulté le 2 février 2012.

MARÉCHAL Aurélie, 2011. "Économie écologique : principes de base ", dans Etopia n 8 , http://www.etopia.be/spip.php?article1741, consulté le 28 octobre 2011.

DIEMER Arnaud et al., 2007. "L'écologie industrielle : quand l'écosystème industriel devient un vecteur du développement durable ", dans Développement durable et territoires, http://developpementdurable.revues.org/412, consulté le 2 novembre 2012.

MARTíNEZ ALIER Joan, 2009. " Hacia un decrecimiento sostenible en las economías ricas ", dans Revista de economía crítica, n⿳0 8, http://www.ecologiaradical.com.mx/VB/Biblioteca/Hacia un Decrecimiento Sostenible en las Economías Ricas.pdf, consulté le 10 septembre 2011.

TEEB, 2008. L'économie des écosystèmes et de la biodiversité : rapport de mi-parcours, http://www.teebweb.org/Portals/25/Documents/TEEB\%20for\%20Business/TEEB\%20for\%20B us\%20Exec\%20French.pdf, consulté le 23 février 2012.

CEECEC, 2010. Le manuel du CEECEC : l'économie écologique de bas en haut, http://www.ceecec.net/wp-content/uploads/2010/10/FRENCH-HANDBOOK-FINAL.pdf, consulté le 20 décembre 2011.

DUCHEMIN Eric, 2001. "Mathis Wackernagel et William Rees, notre empreinte écologique ", dans Vertigo, http://vertigo.revues.org/5230, consulté le 4 novembre 2012. 